\title{
Erratum
}

\section{Erratum: Sela et al., "Sleep Differentially Affects Early and Late Neuronal Responses to Sounds in Auditory and Perirhinal Cortices"}

In the article "Sleep Differentially Affects Early and Late Neuronal Responses to Sounds in Auditory and Perirhinal Cortices," by Yaniv Sela, Aaron Joseph Krom, Lottem Bergman, Noa Regev, and Yuval Nir, which appeared on pages 2895-2905 of the April 1, 2020 issue, there was a labeling error in Figure $6 D$ in which "early" and "late" were in switched positions. The corrected figure appears below. This error does not affect the conclusions of the paper, and the online version has been corrected.
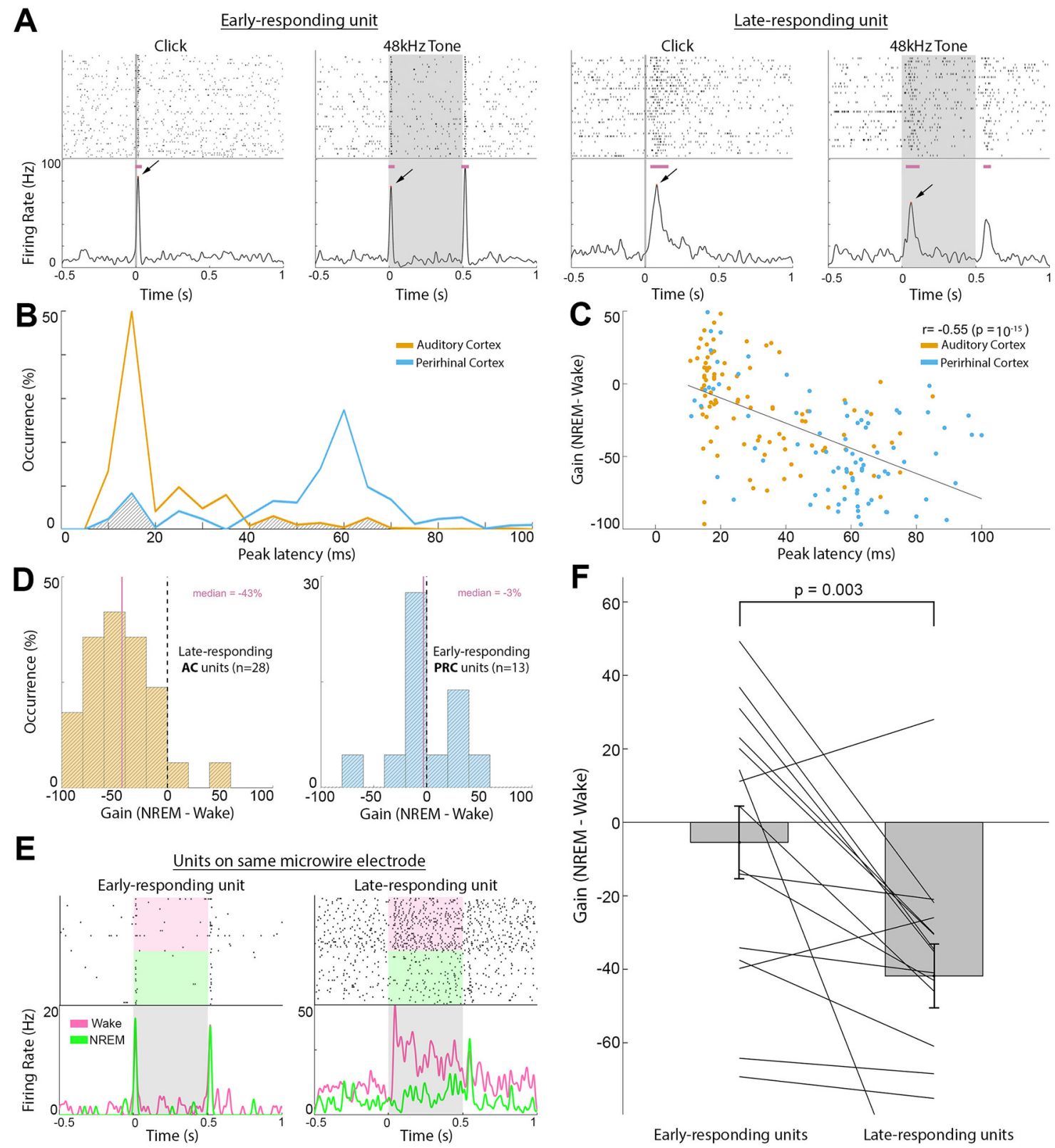

Figure 6. 